\title{
Composição e riqueza de anfíbios anuros em remanescentes de Cerrado do Brasil Central
}

\author{
Priscilla G. Gambale ${ }^{1,2}$, Manoela Woitovicz-Cardoso ${ }^{3}$, Raísa R. Vieira ${ }^{2,4}$, Vinícius G. Batista ${ }^{1,2}$, \\ Jade $\operatorname{Ramos}^{4} \&$ Rogério P. Bastos ${ }^{2}$
}

\footnotetext{
1. Programa de Pós-Graduação em Ecologia de Ambientes Aquáticos Continentais, Universidade Estadual de Maringá, Nupélia - Núcleo de Pesquisas em Limnologia, Ictiologia e Aquicultura, Av. Colombo, 5790, Bloco G-90, 87020-900 Maringá, PR, Brasil. (priscillagambale@gmail.com; vinicius.guerra_@hotmail.com)

2. Laboratório de Herpetologia e Comportamento Animal, Departamento de Ecologia, Instituto de Ciências Biológicas, Universidade Federal de Goiás, ICB 1, Caixa Postal 131, Campus

Samambaia, Setor Itatiaia, 74001-970 Goiânia, GO, Brasil. (rogerioiscinax@gmail.com)

3. Museu Nacional, Universidade Federal do Rio de Janeiro, Departamento de Vertebrados, Quinta da Boa Vista, São Cristóvão, 20940-040 Rio de Janeiro, RJ, Brasil. (manoelawcardoso@gmail.com)

4. Programa de Pós-Graduação em Ecologia e Evolução, Universidade Federal de Goiás, Departamento de Ecologia/Instituto de Ciências Biológicas. Caixa Postal 131, 74001-970, Goiânia, GO, Brasil. (raisa.rsv@gmail.com; jaderamos.bio@gmail.com)
}

ABSTRACT. Anuran richness and composition in remnants of Cerrado of Central Brazil. The biological diversity and ecological processes in Cerrado biome are still poorly understood. Herein we present information about anuran species of the municipality of Barro Alto, state of Goiás (Central Brazil). Five water bodies were sampled between the years 2007 and 2010 (October-March), three of them associated with forested area and two with open areas. We recorded 39 anuran species, characterized as open habitat specialists, forest habitat specialists and generalists. The rarefaction curve shows an asymptote formation, which demonstrates adequate effort and sampling techniques to assess the species richness of the region. Species richness was influenced by air humidity and temperature, since most of the species reproduce in the rainy season. We found significant differences in species composition between breeding sites in open and forested areas. The anurofauna in Cerrado seems to be formed from habitat's horizontal heterogeneity in space. Thus, contrasting habitats in space are important for maintaining the anuran richness, and therefore relevant in conservation terms.

KEYWORDS. Amphibia, Anura, assemblage, spatial distribution.

RESUMO. A diversidade biológica e os processos ecológicos do bioma Cerrado ainda são pouco conhecidos. Neste estudo são apresentadas informações acerca da estrutura da anurofauna do município de Barro Alto, estado de Goiás (Brasil Central). Cinco corpos d'água foram amostrados entre os anos de 2007 a 2010 (outubro-março), três deles associados à área florestal e dois associados à área aberta. Registraram-se 39 espécies de anuros, caracterizados como especialistas de hábitat aberto, florestal ou generalistas. A curva de acumulação de espécies apresentou a formação de uma assíntota, evidenciando que as técnicas e esforço de amostragens foram adequados para se estimar a riqueza de espécies de anuros na região. A riqueza foi influenciada pela umidade e temperatura, já que a maioria das espécies se reproduz na estação quente e chuvosa. Diferenças significativas na composição de espécies entre os sítios reprodutivos de áreas florestais e abertas foram registradas. A fauna de anuros no Cerrado parece ser constituída a partir da heterogeneidade horizontal dos hábitats no espaço. Assim, hábitats contrastantes no espaço são importantes para a manutenção da riqueza da anurofauna, e portanto, relevantes em termos conservacionistas.

PALAVRAS-CHAVE. Amphibia, Anura, taxocenose, distribuição espacial.

O Cerrado é o segundo maior bioma fitogeográfico brasileiro e um dos 34 hotspots de biodiversidade, com elevado número de espécies endêmicas que caracterizam a região como prioritária para estudos conservacionistas (Mittermeier et al., 2004). Avanços nas pesquisas têm sido evidenciados, mas a diversidade biológica e processos ecológicos do Cerrado ainda são pouco conhecidos (Colli et al., 2002; Brites et al., 2009; Tonial et al., 2012). Estudos no bioma são de suma importância, pois a intensa ação antrópica, promovida principalmente através das expansões agrícolas, tem modificado ecossistemas e os hábitats naturais (KLINK \& MACHADO, 2005). Com isso, muitas espécies estão sendo extintas sem ao menos serem formalmente descritas (Pombal \& Gordo, 2004; DinIZFiLHo et al., 2009).

Recentemente, tem ocorrido aumento no número de pesquisas sobre as assembleias de anuros nas regiões interioranas do Cerrado (Uetanabaro et al., 2007; GiaretTA et al., 2008; Oda et al., 2009; Kopp et al., 2010; Morais et al., 2011; Nomura et al., 2012; Morais et al., 2012; CAMPOS et al., 2013). Todavia, o conhecimento básico sobre tais comunidades ainda é incipiente, sendo importante estudos sobre a composição de anuros em áreas com diferentes níveis de variação ambiental (Nomura et al., 2012; CAMPOS et al., 2013), sobre diferenças na diversidade de espécies entre regiões (Tonial et al., 2012), sobre a distribuição geográfica real (MorAis et al., 2011) e a diversidade local das espécies (Oda et al., 2009; Valdujo et al., 2009). Atualmente, são conhecidas 209 espécies de anfíbios anuros para o bioma, sendo 108 consideradas endêmicas (VALDUJo et al., 2012). Segundo VALDujo et al. (2011) diferenças na composição de espécies entre localidades são resultados de fatores históricos que contribuem para a distribuição diferencial de cada espécie.

A alta riqueza da herpetofauna no Cerrado tem sido atribuída à heterogeneidade ambiental, criada a partir de um mosaico de hábitats horizontalmente contrastantes no espaço (e.g. hábitats abertos, florestais, secos e úmidos) que disponibiliza um alto número de recursos para as espécies (Colli et al., 2002; Nogueira et al., 2005; Nogueira et 
al., 2009). Assim, não só os ambientes florestais, mas também áreas abertas tropicais, são importantes para a manutenção da riqueza de espécies (Nogueira et al., 2009), pois garantem que os nichos inerentes a cada organismo sejam mantidos no espaço (CAmpos et al., 2013).

Diante deste cenário, este trabalho tem o objetivo de contribuir para o conhecimento da biodiversidade da anurofauna em uma região do bioma Cerrado. Neste trabalho apresentamos a riqueza e composição de espécies de anuros no município de Barro Alto, estado de Goiás. Adicionalmente, foi feita uma comparação da composição e distribuição dos anuros em cinco sítios reprodutivos, sendo dois inseridos em uma área aberta e três associados a área florestal.

\section{MATERIAL E MÉTODOS}

Área de estudo. $O$ estudo foi realizado no município

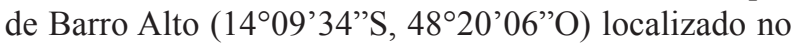
Vale do rio Patrício, região centro-norte do estado de Goiás (GO) (Fig. 1). A região é caracterizada por grande quantidade de rochas vulcano-sedimentares e ao conjunto litológico associado aos complexos máficos-ultramáficos e aos maciços ultramáficos tipo Americano do Brasil (Oliveira et al., 2009).

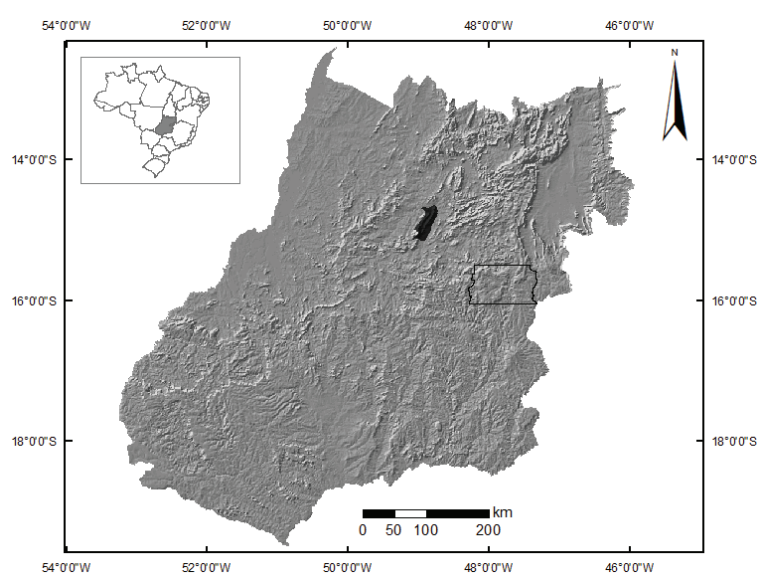

Fig. 1. Localização do município de Barro Alto, GO, Brasil.

A vegetação na região é caracterizada por diferentes fitofisionomias do Cerrado incluindo uma gradação de ambientes savânicos (Cerrado sensu stricto, Campo sujo, Campo de murundus) e florestais (Cerradão), com regiões bem preservadas em elevações acima de 1.000 metros, e com características atípicas em menores elevações associadas à extração mineral e à agricultura (Melfi, 1974). A região é dominada por pastagens com distribuição de arbustos e árvores de pequeno porte (Campo sujo), árvores e arbustos de grande porte com vegetação herbácea entre elas (Cerrado sensu stricto) e regiões composta por árvores que cobrem até 90\% da área (Cerradão) (Oliveira-Filho \& RATTER, 2002).

O clima local é considerado tropical com a presença de duas estações definidas (verão úmido e inverno seco), classificados como tipo Aw no sistema de classificação de Köppen (PeEL et al., 2007). A estação seca, nos meses mais frios, estende-se de maio a setembro e a chuvosa, nos meses mais quentes, de outubro a abril, com temperatura média anual entre $27 \mathrm{e} 31^{\circ} \mathrm{C}$ (GonçALVEs et al., 2013). A maioria das chuvas ocorre durante os meses de novembro a março, com precipitação média anual de $1.400 \mathrm{~mm}$ (Silvando et al., 2006; GonÇALVES et al., 2013).

Hábitats amostrados. Foram selecionados cinco corpos d'água que se apresentaram como hábitats disponíveis para a reprodução de anuros. Dois dos sítios reprodutivos situam-se em áreas abertas, associados a plantações ou pastagens, com remanescentes de formações vegetais do tipo Cerrado sensu stricto e Campo sujo. Os outros três corpos d'água foram associados à área florestal, dominados por formações vegetais do tipo Cerradão e Campo de murundus próximo a veredas. Os locais amostrados constam na Tab. I.

Amostragens. As amostragens de anuros foram realizadas mensalmente entre os meses de outubro a março, durante as estações reprodutivas de 2007 e 2009. $\mathrm{Na}$ estação de 2010 apenas os meses de janeiro a abril foram amostrados, totalizando 16 visitas a campo, que apresentavam em média 10 dias e resultaram em 151 dias de observações. Para padronização do esforço amostral, cada corpo d'água foi percorrido por um coletor durante uma hora, resultando em um esforço de 151 horas/coletor. As amostragens foram realizadas no período noturno entre 18:00 e 01:00, utilizando os métodos de procuras visuais (Crump \& Scott, 1994) e auditivas (Heyer et al., 1994). Para auxiliar na identificação das espécies, as vocalizações foram gravadas e imagens depositadas no Laboratório de Herpetologia e Comportamento Animal da Universidade Federal de Goiás.

Exemplares testemunhos foram coletados, mortos com lidocaína a 5\%, fixados em solução de formalina a $10 \%$

Tab. I. Sítios de amostragem visitados durante o monitoramento de anfíbios anuros no município de Barro Alto, estado de Goiás, Brasil, com os respectivos ambientes em que estão inseridos (florestais ou abertos), fitofisionomia e coordenadas geográficas.

\begin{tabular}{llll}
\hline Ponto & Ambiente & Fitofisionomia & Coordenadas \\
\hline PTN 01 & Vereda & Campo de murundus & $15^{\circ} 05^{\prime} 48^{\prime \prime S}, 49^{\circ} 02^{\prime 2} 22^{\prime \prime} \mathrm{W}$ \\
PTN 02 & Lagoa formada por vereda represada & Cerradão & $15^{\circ} 04^{\prime} 16^{\prime \prime} \mathrm{S}, 48^{\circ} 54^{\prime} 22^{\prime \prime} \mathrm{W}$ \\
PTN 03 & Lagoa & Cerradão & $15^{\circ} 04^{\prime} 59^{\prime \prime S}, 48^{\circ} 59^{\prime} 28^{\prime \prime} \mathrm{W}$ \\
PTN 04 & Lagoa cercada por canavial & Cerrado sensu stricto & $15^{\circ} 03^{\prime} 49^{\prime \prime S}, 48^{\circ} 53^{\prime} 34^{\prime \prime W}$ \\
PTN 05 & Corpo d'água temporário em área de pastagem & Campo sujo & $15^{\circ} 04^{\prime} 18^{\prime \prime S}, 48^{\circ} 54^{\prime} 22^{\prime \prime} \mathrm{W}$ \\
\hline
\end{tabular}


Tab. II. Lista das espécies de anuros registrados durante o monitoramento no município de Barro Alto, estado de Goiás, Brasil, nas estações reprodutivas (outubro de 2007 a março de 2008, outubro de 2009 a março de 2010 e janeiro a abril de 2011). Para cada espécie são observados valores de desvio padrão (DP) e P obtidos através do teste de Monte Carlo. Valores de $\mathrm{P}<0,01$ representam espécies importantes para a formação de grupos de áreas florestais e abertas $(*$, espécies registradas apenas por encontro ocasional em outros corpos d'água).

\begin{tabular}{|c|c|c|c|c|c|c|c|}
\hline & \multicolumn{5}{|c|}{ Sítios reprodutivos amostrados } & \multirow[t]{3}{*}{ DP } & \multirow[t]{3}{*}{$\mathrm{P}$} \\
\hline & \multicolumn{3}{|c|}{ Área florestal } & \multicolumn{2}{|c|}{ Área aberta } & & \\
\hline & PTN 01 & PTN 02 & PTN 03 & PTN 04 & PTN 05 & & \\
\hline \multicolumn{8}{|l|}{ BUFONIDAE } \\
\hline Rhinella mirandaribeiroi (Gallardo, 1965) & & & & $X$ & & 0,77 & 0,38 \\
\hline R. schneideri (Werner, 1894) & $\mathrm{X}$ & $\mathrm{X}$ & & & $\mathrm{X}$ & 2,46 & 1,0 \\
\hline \multicolumn{8}{|l|}{ CRAUGASTORIDAE } \\
\hline Barycholos ternetzi (Miranda-Ribeiro, 1937) & $\mathrm{X}$ & $\mathrm{X}$ & $\mathrm{X}$ & & & 4,25 & $<0,01$ \\
\hline \multicolumn{8}{|l|}{ HYLIDAE } \\
\hline Dendropsophus cruzi (Pombal \& Bastos, 1998) & $\mathrm{X}$ & $\mathrm{X}$ & $\mathrm{X}$ & $\mathrm{X}$ & $\mathrm{X}$ & 3,79 & $\mathbf{0 , 0 2}$ \\
\hline D. rubicundulus (Reinhardt \& Lütken, 1862) & $\mathrm{X}$ & & & $\mathrm{X}$ & $\mathrm{X}$ & 4,41 & 0,32 \\
\hline D. minutus (Peters, 1872) & $\mathrm{X}$ & $\mathrm{X}$ & $\mathrm{X}$ & $\mathrm{X}$ & $\mathrm{X}$ & 3,77 & $<0,01$ \\
\hline D. nanus (Boulenger, 1889) & & $\mathrm{X}$ & & $\mathrm{X}$ & $\mathrm{X}$ & 4,27 & $<0,01$ \\
\hline D. soaresi (Caramaschi \& Jim, 1983) & & & $\mathrm{X}$ & & $\mathrm{X}$ & 1,78 & 1,0 \\
\hline Hypsiboas albopunctatus (Spix, 1824) & $\mathrm{X}$ & $\mathrm{X}$ & $\mathrm{X}$ & $\mathrm{X}$ & $\mathrm{X}$ & 4,33 & $<0,01$ \\
\hline H. lundii (Burmeister, 1856) & $\mathrm{X}$ & & $\mathrm{X}$ & $\mathrm{X}$ & $\mathrm{X}$ & 4,32 & $<\mathbf{0 , 0 1}$ \\
\hline H. paranaiba Carvalho et al., 2010 & $\mathrm{X}$ & $\mathrm{X}$ & $\mathrm{X}$ & $\mathrm{X}$ & & 4,55 & 0,20 \\
\hline H. raniceps Cope, 1862 & $\mathrm{X}$ & $\mathrm{X}$ & & $\mathrm{X}$ & $\mathrm{X}$ & 4,19 & $<0,01$ \\
\hline Phyllomedusa azurea (Cope, 1862) & $\mathrm{X}$ & $\mathrm{X}$ & $\mathrm{X}$ & $\mathrm{X}$ & $\mathrm{X}$ & 4,25 & $<0,01$ \\
\hline Pseudis bolbodactyla (A. Lutz, 1925) & & & $\mathrm{X}$ & $\mathrm{X}$ & $\mathrm{X}$ & 3,98 & 0,37 \\
\hline Scinax aff. fuscomarginatus (A. Lutz, 1925) & $\mathrm{X}$ & $\mathrm{X}$ & & $\mathrm{X}$ & & 3,06 & 1,0 \\
\hline S. constrictus Lima et al., 2004 & & $\mathrm{X}$ & & $\mathrm{X}$ & $\mathrm{X}$ & 4,07 & 0,79 \\
\hline S. fuscomarginatus (A. Lutz, 1925) & $\mathrm{X}$ & $\mathrm{X}$ & & $\mathrm{X}$ & $\mathrm{X}$ & 4,29 & 0,80 \\
\hline S. aff. fuscovarius (A. Lutz, 1925) & $\mathrm{X}$ & $\mathrm{X}$ & & $\mathrm{X}$ & $\mathrm{X}$ & 4,14 & $<0,01$ \\
\hline S. gr. catharinae & $\mathrm{X}$ & $\mathrm{X}$ & & $\mathrm{X}$ & $\mathrm{X}$ & 3,39 & $\mathbf{0 , 0 2}$ \\
\hline Trachycephalus typhonius (Linnaeus, 1758) & & $\mathrm{X}$ & & & $\mathrm{X}$ & 1,78 & 1,0 \\
\hline \multicolumn{8}{|l|}{ LEPTODACTYLIDAE } \\
\hline Adenomera hylaedactyla (Cope, 1868) & $\mathrm{X}$ & $\mathrm{X}$ & $\mathrm{X}$ & & $\mathrm{X}$ & 3,72 & 0,08 \\
\hline A. aff. marmorata Steindachner, 1867 & & & & $\mathrm{X}$ & & 0,76 & 0,36 \\
\hline Leptodactylus fuscus (Schneider, 1799) & $\mathrm{X}$ & $\mathrm{X}$ & & $\mathrm{X}$ & $\mathrm{X}$ & 4,34 & $\mathbf{0 , 0 3}$ \\
\hline L. cf. furnarius Sazima \& Bokermann, 1978 & $\mathrm{X}$ & & & $\mathrm{X}$ & & 2,43 & 1,0 \\
\hline L. labyrinthicus (Spix, 1824) & $\mathrm{X}$ & $\mathrm{X}$ & $\mathrm{X}$ & $\mathrm{X}$ & & 4,06 & $<0,01$ \\
\hline L. aff. latrans (Steffen, 1815) & $\mathrm{X}$ & $\mathrm{X}$ & & $\mathrm{X}$ & $\mathrm{X}$ & 4,02 & $<0,01$ \\
\hline \multicolumn{8}{|l|}{${ }^{*} L$. aff. leptodactyloides (Andersson, 1945) } \\
\hline L. mystaceus (Spix, 1824) & & & & $X$ & $X$ & 2,45 & $\mathbf{0 , 0 2}$ \\
\hline L. mystacinus (Burmeister, 1861) & & & & & $\mathrm{X}$ & 0,76 & 0,36 \\
\hline L. podicipinus (Cope, 1862) & $\mathrm{X}$ & $\mathrm{X}$ & & $\mathrm{X}$ & & 3,70 & 0,08 \\
\hline L. syphax Bokermann, 1969 & $\mathrm{X}$ & & & & $\mathrm{X}$ & 2,41 & 1,0 \\
\hline Physalaemus centralis Bokermann, 1962 & $\mathrm{X}$ & & & $\mathrm{X}$ & $\mathrm{X}$ & 3,36 & $<0,01$ \\
\hline P. cuvieri Fitzinger, 1826 & $\mathrm{X}$ & $\mathrm{X}$ & $\mathrm{X}$ & $\mathrm{X}$ & $\mathrm{X}$ & 3,28 & 0,55 \\
\hline P. nattereri Steindachner, 1863 & $\mathrm{X}$ & & & $\mathrm{X}$ & $\mathrm{X}$ & 3,0 & 0,19 \\
\hline \multicolumn{8}{|l|}{ *Physalaemus $\mathrm{sp}}$. \\
\hline Pseudopaludicola sp. & $\mathrm{X}$ & $\mathrm{X}$ & & $\mathrm{X}$ & $\mathrm{X}$ & 4,25 & $\mathbf{0 , 0 1}$ \\
\hline \multicolumn{8}{|l|}{ MICROHYLIDAE } \\
\hline \multicolumn{8}{|l|}{ *Chiasmocleis albopunctata (Boettger, 1885) } \\
\hline Elachistocleis cf. cesarii (M.-Ribeiro, 1920) & $\mathrm{X}$ & & & $\mathrm{X}$ & $\mathrm{X}$ & 3,9 & $<0,01$ \\
\hline \multicolumn{8}{|l|}{ ODONTOPHRYNIDAE } \\
\hline Proceratophrys bagnoi Brandão et al., 2013 & $\mathrm{X}$ & & & & & 0,76 & 1,0 \\
\hline Riqueza de espécies & 27 & 22 & 12 & 28 & 27 & & \\
\hline Riqueza de espécies por área & & 32 & & & & & \\
\hline
\end{tabular}


e, posteriormente, conservados em álcool 70\%. O material esta depositado na Coleção Zoológica da Universidade Federal de Goiás (ZUFG). A nomenclatura das espécies está de acordo com Frost (2014).

Análise de dados. Para avaliar a riqueza de anuros, foram confeccionadas curvas de riqueza estimada e acumulada de espécies (Gotelli \& Colwell, 2001) através do programa EstimateS 8.0 (Colwell, 2005) a partir de 1000 aleatorizações (Colwell \& Coddington, 1994). A riqueza foi estimada a partir do estimador Jacknife de primeira ordem, por ser um método não-paramétrico que oferece uma estimativa mais acurada da comunidade e produz intervalos de confiança com base nas espécies raras (KREBS, 1999). Tal estimador foi construído a fim de reduzir o viés de tamanho das amostras e projetar o número real de espécies em um dado hábitat amostrado (Rosenzweig et al., 2003). Também foi realizada uma rarefação padronizada por amostras pela técnica Mao Tau (Gotelli \& ColWell, 2001), que permite comparações de riquezas entre amostras e elimina a necessidade de réplicas (Colwell et al., 2004). Cada dia de visita em um corpo d'água foi considerado uma unidade amostral, resultando em 151 amostras ao final das coletas.

Dados sobre as variáveis climáticas - pluviosidade total $(\mathrm{mm})$, umidade relativa do ar (\%), temperaturas máxima e mínima $\left({ }^{\circ} \mathrm{C}\right)$ e número de dias de chuva durante o mês foram obtidas no site da Agência Nacional das Águas (http://www.ana.gov.br/, 2014). Para avaliar possíveis associações da riqueza de espécies registradas nos corpos d'água amostrados com as variáveis climáticas, regressões múltiplas foram conduzidas sendo considerados significativos valores de $\mathrm{P}<0,05$ (ZAR, 1999). As regressões múltiplas foram realizadas no programa Statistica 7.0.

Para descrever a organização espacial da composição de espécies entre os corpos d'água amostrados, foram construídas Ordenações de Escalonamento Multidimensional Não-métrico (NMDS), através da medida de distância de Jaccard (MANLY, 1994). A distorção da ordenação em relação à matriz de dados originais foi determinada pelo "stress". Segundo RoHLF (2000), valores do "stress" em torno de 0,2 correspondem a ajuste regular, enquanto que em torno de 0,1 indicam um bom ajuste e valor igual a 0 um ajuste perfeito. Posteriormente, resultados do teste de Monte Carlo após 5000 aleatorizações verificaram quais espécies mais contribuíram para a formação dos grupos na NMDS. Uma vez identificados os grupos, análises multivariadas de variância permutacional (PERMANOVA) avaliaram a existência de diferenças significativas entre os corpos d'água amostrados, entre as áreas e entre os meses e anos de amostragem. O nível de significância utilizado foi de $\mathrm{P}<0,05$. As análises foram realizadas através do programa "R for Windows" (R Project For Statistical Computing, 2009).

\section{RESULTADOS}

Foram registradas 39 espécies de anfíbios anuros pertencentes a seis famílias e 15 gêneros. Hylidae apresentou maior número de espécies $(\mathrm{N}=17 ; 43,6 \%)$, seguida por Leptodactylidae $(\mathrm{N}=16 ; 41 \%)$, Microhylidae e Bufonidae $(\mathrm{N}=2$, cada; $5,1 \%)$. Odontophrynidae e Craugastoridae apresentaram apenas uma espécie cada $(2,6 \%$, cada) (Tab. II). Indivíduos de Physalaemus sp., Leptodactylus cf. leptodactyloides e Chiasmocleis albopunctata foram registrados apenas ocasionalmente durante uma, duas e cinco noites de observações respectivamente.

A curva de rarefação de espécies tende a uma assíntota a partir da amostra 90 , pois não há diferenças entre os intervalos de confiança que se sobrepõem entre a riqueza observada e estimada, o que explica que o esforço amostral foi suficiente para representar as espécies desta comunidade (Fig. 2). As 39 espécies registradas para o município representam $92,8 \%$ da riqueza teórica estimada pelo estimador Jackknife de primeira ordem (42 espécies). A riqueza observada nos sítios reprodutivos amostrados foi correlacionada positivamente com a temperatura máxima e umidade relativa do ar (Tab. III).

Os corpos d'água selecionados apresentaram composição de espécies diferentes entre si. Nos sítios relacionados a áreas florestais, foram registradas 32 espécies (27 spp. - PTN 01, 22 spp. - PTN 02, 12 spp. - PTN 03) sendo duas exclusivas deste tipo de ambiente: Barycholos ternetzi e Proceratophrys bagnoi. Nos sítios relacionados

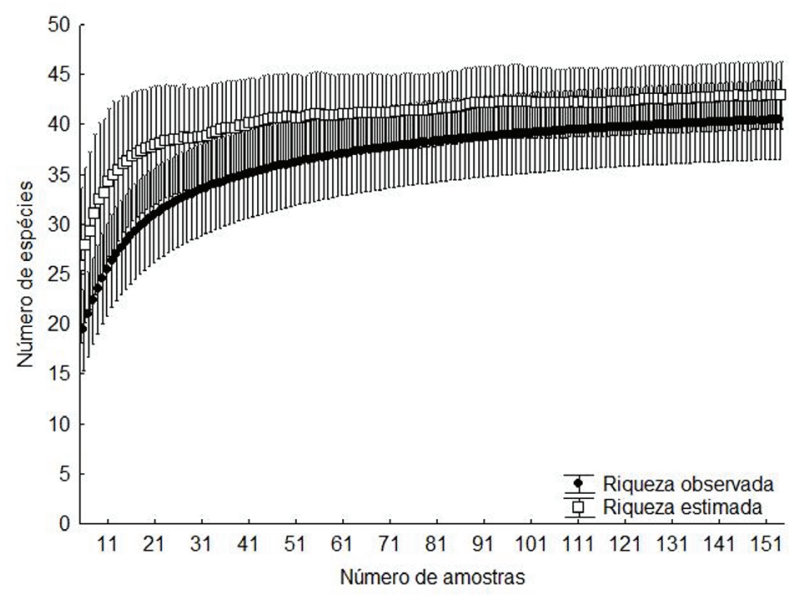

Fig. 2. Curvas de rarefação de espécies nas áreas amostradas, Barro Alto, estado de Goiás, Brasil.

Tab. III. Regressão múltipla da pluviosidade (mm), número de dias de chuva no mês, temperatura máxima e mínima $\left({ }^{\circ} \mathrm{C}\right)$ e umidade relativa do ar (\%) com a riqueza observada nos corpos d'água amostrados. $\beta$ representa o valor de beta referente a regressão múltipla. Valores significativos em negrito $(\mathrm{P}<0,05)$.

\begin{tabular}{ll}
$\beta$ da pluviosidade & 0,001 \\
$\beta$ de dias de chuva & $-0,001$ \\
$\beta$ de temperatura máxima & $\mathbf{0 , 0 9}$ \\
$\beta$ de temperatura mínima & 0,09 \\
$\beta$ de umidade & $\mathbf{0 , 0 3}$ \\
$r^{2}$ aj. & 0,81 \\
$F$ & 78,56 \\
\hline
\end{tabular}


ao ambiente aberto, foram registradas 34 espécies (27 spp. - PTN 04, 28 spp. - PTN 05), sendo quatro espécies exclusivas deste tipo de ambiente: Rhinella mirandaribeiroi, Adenomera aff. marmorata, L. mystacinus e L. mystaceus.

Considerando a composição de espécies, grupos foram formados para cada sítio reprodutivo e também para área florestal e aberta (NMDS, stress =0,194, Fig. 3). Houve diferenças na composição das assembleias de anuros entre os corpos d'água $(\mathrm{F}=17,719 ; \mathrm{P}<0,001)$ e entre as áreas $(\mathrm{F}=16,35 ; \mathrm{P}<0,001)$. Não foram registradas diferenças nas composições da anurofauna entre os meses $(\mathrm{F}=0,848, \mathrm{P}=0,692)$ e anos de amostragem $(\mathrm{F}=0,382$; $\mathrm{P}=0,128)$. E, por fim, verificou-se que algumas espécies influenciaram a formação do grupo correspondente à área florestal (Hypsiboas lundiii, H. albopunctatus, Barycholos ternetzi e Leptodactylus labyrinthicus), enquanto outras influenciaram a formação do grupo correspondente à área aberta (Dendropsophus cruzi, D. rubicundulus, D. nanus, Elachistocleis cf. cesarii, H. raniceps, L. mystaceus, Leptodactylus aff. latrans, Phyllomedusa azurea, Physalaemus centralis, Pseudopaludicola sp., Scinax aff. fuscovarius e Scinax gr. catharinae) (Tab. II, Fig. 4).

\section{DISCUSSÃO}

A riqueza local registrada (39 espécies) para o município de Barro Alto é similar quando comparada a outras regiões amostradas no Cerrado Central: 47 espécies na bacia do rio Caiapó (SILVA JR. et al., 2007), 38 em Niquelândia (ODA et al., 2009; NomUra et al., 2012), 33 no vale do alto rio Tocantins (SILVA JR. et al., 2005), 32 na UHE Espora, estado de Goiás (VAz-SiLVA et al., 2007), 32 em São Desidério, Chapadão Ocidental da Bahia (VAldujo et al., 2009), 25 no Parque Nacional das Emas (Kopp et al., 2010), 22 em Hidrolândia (CAmpo \&VAz-Silva, 2010), 36 no sudoeste do estado de Goiás (Morais et al., 2011), 33 na Floresta Nacional de Silvânia (Morais et al., 2012) e 19 espécies em Tangará da Serra, Mato Grosso (CAmpos et al., 2013). A região de Barro Alto é caracterizada por uma elevada complexidade entre hábitats, permitindo uma alta riqueza de espécies, as quais representam $18,66 \%$ do total das 209 com ocorrência no bioma Cerrado (VALDujo et al., 2012).

A maioria das espécies registrada na região é amplamente distribuída na América do Sul (29 spp.; 74,3\%), sendo 9,3\% consideradas endêmicas do Cerrado: Barycholos ternetzi, Chiasmocleis albopunctata, Dendropsophus cruzi, Dendropsophus rubicundulus, Hypsiboas lundii, Phyllomedusa azurea, Physalaemus centralis, P. nattereri, Rhinella mirandaribeiroi e Scinax constrictus (VALDUJo et al., 2012). O predomínio de espécies com ampla distribuição, complementado pela ocorrência de espécies endêmicas desse bioma é corroborado em outros estudos conduzidos no Cerrado (VAldujo et al., 2009, 2011; Morais et al., 2011).

A estabilidade da curva de acumulação de espécies, bem como o estimador de riqueza da área, evidenciam que as técnicas de amostragens foram apropriadas para registrar a riqueza de anuros na região. Desta forma, nossos dados confirmam que as amostragens realizadas nos sítios reprodutivos são eficientes, pois a ecologia reprodutiva da maioria de anuros registrados os torna dependentes de corpos d'água lênticos ou lóticos (SANTos et al., 2007). Adicionalmente, estudos a longo prazo e amostragens em diferentes tipos de ambientes resultam em uma melhor estimativa da riqueza total de uma determinada área (CECHIN \& MARTins, 2000), favorecendo a ampliação de distribuição de alguns táxons e descoberta de novas espécies (e.g. Caramaschi, 2010; Pombal et al., 2011), bem como a compreensão dos padrões regionais de riqueza das espécies (VALDujo et al., 2011).

Fatores abióticos como umidade, temperatura e pluviosidade são importantes para anfíbios anuros, visto que são organismos ectotérmicos e com pele permeável (Duellman \& Trueb, 1994). Assim, muitos estudos têm evidenciado a influência de tais parâmetros sobre a atividade reprodutiva desses organismos (e.g. CONTE \& RosSA- Feres, 2006; SANTOS et al., 2007; FoRTI, 2009; OdA et al., 2009).
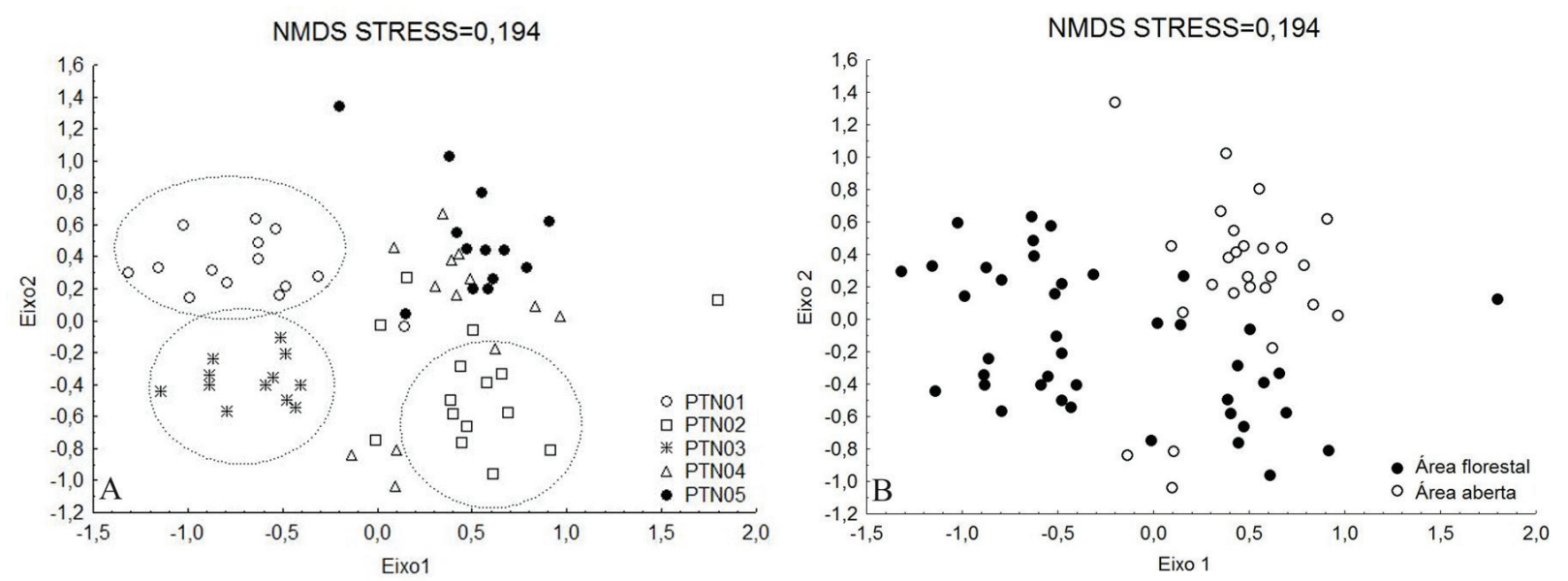

Fig. 3. Gráfico de ordenação por NMDS baseada no índice de Jaccard (dados de presença e ausência) mostrando a dissimilaridade da composição das assembleias de anuros no município de Barro Alto, GO, entre os sítios amostrados (A) e área florestal e aberta (B) (stress=0,194). 


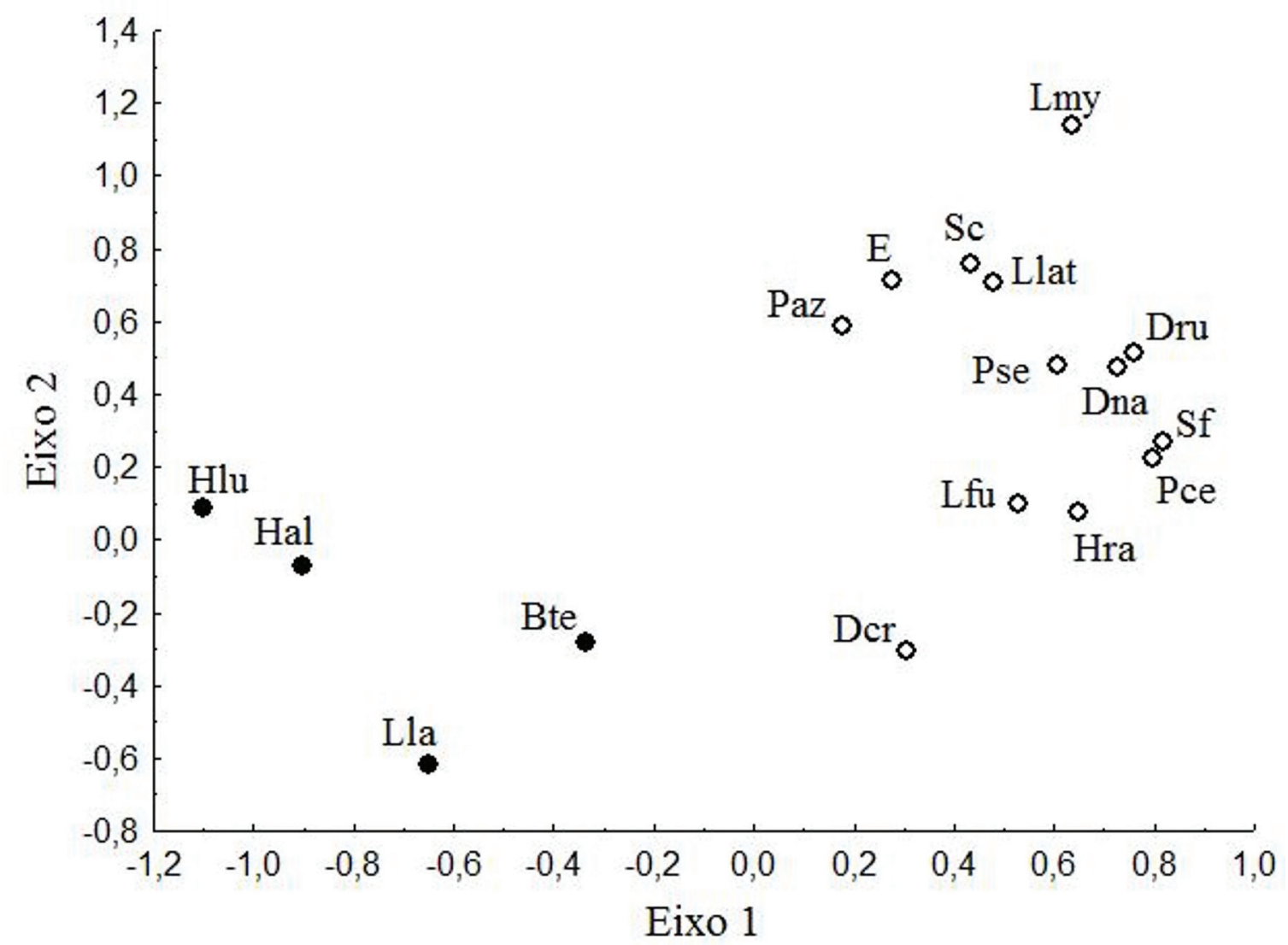

Fig. 4. Gráfico de ordenação por NMDS baseada no índice de Jaccard (dados de presença e ausência) da ocorrência das espécies de anuros registradas para o município de Barro Alto, GO entre as estações reprodutivas de 2007 a 2010 (Stress=0,194) mais importantes para ordenação da área florestal (•) e aberta (০) (Bte, Barycholos ternetzi; Dcr, Dendropsophus cruzi; Dru, D. rubicundulus; Dna, D. nanus; E, Elachistocleis cf. cesarii; Hal, Hypsiboas albopunctatus; Hlu, H. lundii; Hra, H. raniceps; Lfu, L. fuscus; Lla, L. labyrinthicus; Lmy, L. mystaceus; Llat, L. aff. latrans; Paz, Phyllomedusa azurea; Pce, Physalaemus centralis; Pse, Pseudopaludicola sp.; Sf, Scinax aff. fuscovarius; Sc, Scinax sp. (gr. S. catharinae).

A relação entre fatores abióticos e riqueza de espécies no presente trabalho corrobora o registrado anteriormente para ambientes tropicais sazonais, nas quais a maioria das espécies se reproduz na estação quente e chuvosa, como já evidenciado para anuros do Cerrado (Brasileiro et al., 2005; Prado et al., 2005; SANTOS et al., 2007; ForTi, 2009). Sabe-se que temperaturas mais baixas são um indicativo de final de estação chuvosa e, consequentemente, do término da estação reprodutiva da maioria dos anuros (ETEROVICK \& SAZIMA, 2000), assim ocorre uma diminuição da riqueza nos sítios reprodutivos nesse período. Outros fatores como luminosidade e vento também podem influenciar negativamente a atividade dos anuros e consequentemente sua distribuição no ambiente (POMBAL JR., 1997; PRADO \& Pombal, 2005).

A baixa sobreposição entre os sítios reprodutivos e tipos de áreas amostradas (florestal e aberta) é resultado do registro de espécies hábitat especialistas, particularmente àquelas com modos reprodutivos específicos (BERTOLLUCI \& Rodrigues, 2002; Moraes et al., 2007; Oda et al., 2009). Assim, espécies como Barycholos ternetzi, Hypsiboas lundii e Proceratophrys bagnoi podem ser consideradas hábitat especialistas por ter preferência por hábitats florestais para a reprodução. Barycholos ternetzi e P.bagnoi foram registrados somente na área florestal, pois a primeira tem desenvolvimento direto e deposita seus ovos sobre o folhiço úmido da floresta e a segunda utiliza corpos d'água inseridos em tal hábitat para a oviposição (BASTOS et al., 2003; Brasileiro et al., 2005). Hypsiboas lundii, apesar de ter sido registrada nas duas áreas, influenciou a formação do grupo de área florestal devido ao maior número de ocorrências neste ambiente, como registrado em estudos anteriores (BAstos et al., 2003; BrAsileIro et al., 2005; Silva \& GiaretTA, 2009). Todavia, Hypsiboas albopunctatus e Leptodactylus labyrinthicus, que também influenciaram a composição da área florestal, podem ser consideradas generalistas e incidentais nesse hábitat, visto que estavam presentes na mesma proporção nas duas áreas (BRASILEIRO et al., 2005). A segregação espacial, evidenciada para os anuros entre os sítios reprodutivos e os dois tipos de ambiente, corrobora os resultados encontrados em outros trabalhos de distribuição da herpetofauna no Cerrado (Nogueira et al., 2005, 2009; Brasileiro et al., 2005), onde a sobreposição de espécies de répteis e anfíbios entre ambientes abertos e fechados é baixa.

Parte da anurofauna registrada para o município é dominada por especialistas de hábitat aberto, como ficou evidenciado pelo alto número de espécies que influenciaram 
a composição da área aberta no presente estudo. Apesar desta área apresentar alterações antrópicas, características ambientais como maior exposição solar, menor umidade e temperaturas elevadas podem favorecer algumas espécies de anuros que apresentam resistência em relação a perda de água através da superfície corporal (MORAEs et al., 2007). Assim, Rhinella mirandaribeiroi pode ter sido registrada somente na área aberta devido à elevada tolerância à escassez de água (THORson, 1955). Adicionalmente, características ambientais específicas como a presença de fragmentos florestais próximos, disponibilidade de abrigos e de alimento e local adequado para reprodução podem também favorecer a persistência de populações de anuros (Henderson \& Powell, 2001).

Em geral, a anurofauna de Barro Alto apresentou poucas espécies com especificidade em relação aos hábitats reprodutivos (Brasileiro et al., 2005). A ocorrência de espécies mais generalistas pode ser associada à sazonalidade e constantes alterações antrópicas no Cerrado, que tornam os corpos d'água efêmeros, estimulando a colonização de novas áreas por estas espécies (RosSA-Feres \& JIM, 1994).

A fauna de anuros no Cerrado parece ser influenciada pela heterogeneidade horizontal dos hábitats no espaço, ocasionada por diferentes tipos de vegetação (e.g. áreas florestais e abertas). Isto favorece a ocorrência de espécies especialistas, bem como de generalistas, mantendo uma alta diversidade (BRIDGEWATER et al., 2004; NogueIRA et al., 2009), como predito pela hipótese de estratificação horizontal de hábitats (PIANKA, 1966; Colli et al., 2002) e observado no presente trabalho. Assim não só florestas tropicais, mas também áreas abertas tropicais, são essenciais para a manutenção da diversidade de espécies no Cerrado (Colli et al., 2002; Nogueira et al., 2009). Além disso, como ambientes abertos são constantemente negligenciados em termos de esforços de conservação e proteção legal, pode-se concluir que esta falha pode levar à perda de biodiversidade (DinIZ-FilHo et al., 2007).

Agradecimentos. Agradecemos FHO pela leitura crítica e comentários. RPB (processo 304363/2010-3) agradece ao CNPq pela bolsa de produtividade em pesquisa; a CAPES pelas bolsas de mestrado e doutorado concedidas a PGG, RRV e VBG. MWC agradece ao CNPq e à FAPERJ pelo auxílio financeiro. À AngloAmerican Brasil pelo auxílio financeiro e apoio logístico. A Fundação de Apoio a Pesquisa da Universidade Federal de Goiás (FUNAPE/UFG), pelo gerenciamento dos recursos e pela concessão de bolsa a MWC. As licenças de coleta foram fornecidas pelo Instituto Chico Mendes de Conservação da Biodiversidade (ICMBio/RAN).

\section{REFERÊNCIAS BIBLIOGRÁFICAS}

Bastos, R. P.; Motta, J. A. O.; Lima, L. P. \& Guimarães, L. D. 2003. Anfíbios da Floresta Nacional de Silvânia, estado de Goiás. Goiânia, Stylo Gráfica e Editora. 29p.

Bertolluci, J. \& Rodrigues, M. T. 2002.Seasonal patterns of breeding activity of Atlantic Rainforest anurans at Boracéia, Southeastern Brazil. Amphibia-Reptilia 23(2):161-167.

Brasileiro, C. A.; Sawaya, R. J.; Kiefer, M. C. \& Martins, M. 2005. Amphibians of the Cerrado of Itirapina Ecological Station, Southeastern Brazil. Biota Neotropica 5(2):1-17.

Bridgewater, S.; Ratter, J. A. \& Ribeiro, J. F. 2004. Biogeographic patterns, $\beta$-diversity and dominance in the cerrado biome of Brazil. Biodiversity and Conservation 13(12):2295-2318.

Brites, V. L. de C.; Faria, R. G.; Mesquita, D. O. \& Colli, G. R. 2009. The herpetofauna of the neotropical savannas. In: Del Claro, K.; Rico-Gray, V.; Barbosa, A. A. A.; Bonet, A.; Scarano, F. R.; Garzon, F. J. M.; Villarnovo, G. C.; Coelho, L.; Sampaio, M. V.; Quesada, M.; Morris, M. R. \& RamireZ, N. eds. Encyclopedia of Life Support Systems (EOLSS). Oxford, Eolss Publishers. v. X.

CAMPO, F. S. \& VAZ-SiLVA, W. 2010. Distribuição espacial e temporal da anurofauna em diferentes ambientes no município de Hidrolândia, GO, Brasil Central. Neotropical Biology and Conservation 5(3):179-187.

Campos, V. A.; Oda, F. H.; Juen, L.; Barth, A. \& Dartora, A. 2013. Composição e riqueza de espécies de anfíbios anuros em três diferentes hábitats em um agrossistema no Cerrado do Brasil central. Biota Neotropica 13(1):1-9.

CARAmaschi, U. 2010. Notes on the taxonomic status of Elachistocleis ovalis (Schneider, 1799) and description of five new species of Elachistocleis Parker, 1927 (Amphibia, Anura, Microhylidae). Boletim do Museu Nacional, Zoologia 2010(527):1-30.

Céchin, S. Z. \& Martins, M. 2000. Eficiência de armadilhas de queda (pitfall traps) em amostragens de anfibios e répteis no Brasil. Revista Brasileira de Zoologia 17(3):729-740.

Colli, G. R.; Bastos, R. P. \& Araujo, A. F. B. 2002. The character and dynamics of the Cerrado herpetofauna. In: Oliveira, P. S. \& MARQuIs, R. J. eds. The Cerrados of Brazil: Ecology and natural history of neotropical savanna. New York, Columbia University Press. p. 223-241.

Colwell, R. K. 2005. EstimateS: Statistical estimation of species richness and shared species from samples, version 7.5. Disponível em: <www.purl.oclc.org/estimates $>$. Acesso em: 20.03.2013.

Colwell, R. K. \& Coddington, J. A. 1994. Estimating terrestrial biodiversity through extrapolation. Philosophical Transactions of the Royal Society B 345(1311):101-118.

Colwell, R. K.; MAO, C. X. \& ChANG, J. 2004. Interpolating, extrapolating and comparing incidence-based species accumulation curves. Ecology 85(10):2717-2727.

Conte, C. E. \& Rossa-Feres, D. C. 2006. Diversidade e ocorrência temporal da anurofauna (Amphibia, Anura) em São José dos Pinhais, Paraná, Sul do Brasil. Revista Brasileira de Zoologia 23(1):162-175.

CRump, M. \& SCOTT JunioR, N. J. 1994. Standard techniques for Inventory and Monitoring. In: Heyer, W. R.; Donnelly, M. A.; McDiarmid, R. W.; HAYEK, L. A. C. \& Foster, M. S. eds. Measuring and Monitoring Biological Diversity. Washington, London, Smithsonian Institution Press. p.84-92.

Diniz-Filho, J. A. F.; Bini, L. M.; Pinto, M. P.; Rangel, T. F. L. V. B.; Vieira, S. L. \& BASTos, R. P. 2007. Conservation biogeography of anurans in Brazilian Cerrado. Biodiversity and Conservation 16:997-1008.

Diniz-Filho, J. A. F.; Bini, L. M.; Oliveira, G.; Barreto, B. S.; Silva, M. M. F. P.; Terrible, L.; Rangel, T. F. L. V.; Pinto, M. P.; Sousa, N. P. R.; Vieira, L. C. G.; Melo, A. S.; De Marco Junior, P.; Blamires, D.; Bastos, R. P.; Carvalho, P.; Ferreira, L. G.; Telles, M. P. C.; Rodrigues, F. M.; Silva, D. M.; Silva JR., N. J. \& Soares, T. N. 2009. Macroecologia, biogeografia e áreas prioritárias para conservação no cerrado. Oecologia Brasiliensis 13(3):470-497.

Duellman, W. E. \& Trueb, L. 1994. Biology of amphibians. Baltimore, The John Hopkins University Press. 670p.

Eterovick, P. C. \& SAzima, I. 2000. Structure of an anuran community in a montane meadow in southeastern Brazil: effects of seasonality, hábitat, and predation. Amphibia-Reptilia 21(4):439-461.

ForTI, L. R. 2009. Temporada reprodutiva, micro-hábitat e turno de vocalização de anfíbios anuros em lagoa de Floresta Atlântica, no Sudeste do Brasil. Revista Brasileira de Zoociências 11(1):89-98.

Frost, D. R. 2014. Amphibian Species of the world: an online reference, version 6.0. American Museum of Natural History, New York. Disponível em: <http://research.amnh.org/herpetology/amphibia/ index.html>. Acesso em: 08.03.2014.

Giaretta, A. A.; Menin, M.; Facure, K. G.; Kokubum, M. N. C. \& De Oliveira Filho, J. C. 2008. Species richness, relative abundance, and hábitat of reproduction of terrestrial frogs in the Triângulo 
Mineiro region, Cerrado biome, southeastern Brazil. Iheringia, Série Zoologia 98(2):181-188.

Gonçalves, M. W.; Carvalho, W. F.; Da Cruz, A. D.; Vieira, R. R. S.; de Morais, A. R.; Gambale, P. G.; Batista, V. G.; Bastos, R. P. \& SILVA, D. M. 2013. Contrasting Patterns of DNADamage by the Comet Assay in Four Species of the Hylidae Family (Amphibia-Anura). Research Journal of Biology 01(1):1-6.

Gotelli, N. J. \& Colwell, R. K. 2001. Quantifying biodiversity: procedures and pitfalls in the measurement and comparison of species richness. Ecology Letters 4(4):379-391.

Henderson, R. W. \& Powell, R. 2001. Responses by the West Indian herpetofauna to human-influenced resources. Caribbean Journal of Science 37(1-2):41-54.

Heyer, W. R.; Donnelly, M. A.; McDiamird, R. W.; Hayek, L. C. \& FosTER, M. 1994. Measuaring and monitoring biological diversity standard methods for amphibians. Washington D.C., London, Smithsonian Institution Press. 364p.

KLINK, C. A. \& MACHADO, R. B. 2005. A conservação do cerrado brasileiro. Megadiversidade 1(1): 147-155.

Kopp, K.; Signorelli, L. \& Bastos, R. P. 2010. Distribuição temporal e diversidade de modos reprodutivos de anfíbios anuros no Parque Nacional das Emas e entorno, estado de Goiás, Brasil. Iheringia, Série Zoologia 100(3): 192-200.

Krebs, C. J. 1999. Ecological Methodology. Menlo Park, Addison Wesley Educational Publishers. 620p.

Manly, B. F. G. 1994. A Primer of Multivariate Statistics. London, Chapman \& Hall. 179p.

Melfi, A. J. 1974 . Características geoquímicas e mineralógicas dos estágios iniciais da alteração superficial das rochas ultrabásicas de Barro Alto, GO. Boletim IG-USP Série Científica 5:117-128.

Mittermeier, R. A.; Robles, G. P.; Hoffmann, M.; Pilgrim, J.; Brooks, T.; Mittermeier, C. G.; Lamoreux, J. \& Da Fonseca, G. A. B. 2004. Hotspots Revisited. N.L. Mexico, CEMEX. 390p.

Moraes, R. A. DE; Sawaya, R.J.; Barrella, W. 2007. Composição e diversidade de anfíbios anuros em dois ambientes de Mata Atlântica no Parque Estadual Carlos Botelho, SP. Biota Neotropica 7(2):1-10.

Morais, A. R. ; Bastos R. P.; Vieira, R. R. S \& Signorelli, L. 2012. Herpetofauna da Floresta Nacional de Silvânia, um remanescente de Cerrado no Brasil Central. Neotropical Biology and Conservation 7(2):114-121.

Morais, A. R. D.; Signorelli, L.; Gambale, P. G.; Kopp, K.; Guimaraes, L. D.; VAz-SilVA, W.; RAmOS, J. \& BASTOS, R. P. 2011. Anfíbios anuros associados a corpos d'água do sudoeste do estado de Goiás, Brasil. Biota Neotropica 11(3):355-363.

Nogueira, C.; Colli, G. R. \& Martins, M. 2009. Local richness and distribution of the lizard fauna in natural hábitat mosaics of the Brazilian Cerrado. Austral Ecology 34(1):83-96.

Nogueira, C.; Valdujo, P. \& França, F. 2005. Hábitat variation and lizard diversity in a Cerrado area of Central Brazil. Studies on Neotropical Fauna and Environment 40(2):105-112.

Nomura, F.; Maciel, N. M.; Pereira, E. B. \& Bastos, R. P. 2012. Diversidade de anuros (Amphibia) em áreas recuperadas de atividade mineradora no Cerrado e de plantio de Eucalyptus urophyla no Brasil Central. Bioscience Journal 28(2):312-324.

Oda, F. H.; Bastos, R. P. \& SÁ Lima, M. A. D. C. 2009. Taxocenose de anfíbios anuros no Cerrado do Alto Tocantins, Niquelândia, estado de Goiás: diversidade, distribuição local e sazonalidade. Biota Neotropica 9(4):219-232.

Oliveira, F. S.; Varajao, A. F. D. C.; Varajao, C. A. C.; Boulangé, B.; Costa, J. L. G. \& VessanI, L. A. 2009. Alteração supergênica e morfogênese tropical no Complexo Máfico-Ultramáfico Acamadado de Barro Alto, GO. Geociências 28(3):255-272.

Oliveira-Filho, A. T. \& Ratter, J. A. 2002. Vegetation physiognomies and woody flora of the Cerrado Biome. In: OLIVEIRA, P. S. \& MARQuis, R. J. eds. The Cerrados of Brazil: ecology and natural history of a Neotropical savanna. New York, Columbia University Press. p. 91-120.

Peel, M. C.; Finlayson, B. L. \& McMahon, T. A. 2007. Updated world map of the Köppen-Geiger climate classification. Hydrology and Earth System Science 11:1633-1644.

PiANKA, E. R. 1966. Convexity, desert lizards and spatial heterogeneity.
Ecology 47:1055-1059.

Pombal JR., J. P. 1997. Distribuição espacial e temporal de anuros (Amphibia) em uma poça permanente na Serra de Paranapiacaba, sudeste do Brasil. Revista Brasileira de Biologia 57(4):583-594.

Pombal JR., J. P. \& Gordo, M. 2004. Anfíbios anuros da Juréia. In: Marques, O.A. V. \& DulebA, W. eds. Estação Ecológica JuréiaItatins. Ambiente Físico, Flora e Fauna. Ribeirão Preto, Holos. p. 243-256.

Pombal Jr., J. P.; Bilate, M.; Gambale, P. G.; Signorelli, L. \& Bastos, R. P. 2011. A new miniature treefrog of the Scinax ruber clade from Cerrado of Central Brazil (Anura: Hylidae). Herpetologica 67(3):288-299.

Prado, C. P. A.; Uetanabaro, M. \& Haddad, C. F. B. 2005. Breeding activity patterns, reproductive modes, and hábitat use by anurans (Amphibia) in a seasonal environment in the Pantanal, Brazil. Amphibia-Reptilia 26(2):211-221.

Prado, G. M. \& Pombal JR., J. P. 2005. Distribuição espacial e temporal dos anuros em um brejo da Reserva Biológica de Duas Bocas, sudeste do Brasil. Arquivos do Museu Nacional 63(4):685-705.

R Development Core Team. 2009. R: a language and environment for statistical computing. Vienna, R foundation for statistical computing. Disponível em: <http://www.R-project.org>. Acesso em: 14.01.2013.

RohlF, F. J. 2000. NTSYS 2.1: Numerical Taxonomic and Multivariate Analysis System. New York, Exeter Software.

Rosenzweig, M. L.; Turner, W. R.; Cox, J. G. \& Ricketts, H. T. 2003. Estimating Diversity in Unsampled Hábitats of a Biogeographical Province. Conservation Biology 17(3):864-874.

Rossa-Feres, D. C. \& JiM, J. 1994. Distribuição sazonal em comunidades de anfíbios anuros na região de Botucatu, São Paulo. Revista Brasileira de Biologia 54(4):323-334.

Santos, T. G.; Rossa-Feres, D. C. \& Casatti, L. 2007. Diversidade e distribuição espaço-temporal de anuros em região com pronunciada estação seca do sudeste do Brasil. Iheringia, Série Zoologia 97(1):3749.

Silva JR., N. J.; Silva, H. L. R.; Costa, M. C.; Buononato, M. A.; Tonial, M. L. S.; Ribeiro, R. S.; Moreira, L. A. \& Pessoa, A. M. 2007. Avaliação preliminar da fauna silvestre terrestre do vale do rio Caiapó, Goiás: Implicações para a conservação da biodiversidade regional. Estudos 34(11/12):1057-1094.

Silva JR., N. J.; Silva, H. L. R.; Trefaut, M.; Valle, N. C.; Costa, M. C.; Castro, S. R.; Linder, E.; Joahansson, C. \& Sites, J. W. 2005. A fauna do vale do alto rio Tocantins em áreas de usinas hidrelétricas. Estudos 32(1):57-102.

Silva, W. R. DA \& GiaretTA, A. A. 2009 . On the natural history of Leptodactylus syphax and the evolution of reproductive features in the L. pentadactylus species group. Journal of Natural History 43(3-4):191-203.

Silvando, C. da S.; de Santana, N. M. P. \& Pelegrini, J. C. 2006. Caracterização climática do estado de Goiás. Goiânia, Secretaria de Indústria e Comércio, Superintendência de Geologia e Mineração. $133 \mathrm{p}$.

THORson, T. B. 1955. The relationship of water economy to terrestrialism in amphibians. Ecology 36(1):100-115.

Tonial, M. L. S.; Silva, H. L. R.; Tonial, I. J.; Costa, M. C.; Silva JR., N. J. \& DinIZ-Filho, J. 2012. Geographical patterns and partition of turnover and richness components of beta-diversity in faunas forma Tocantins rivervalley. Brazilian Journal of Biology 72(3):497-504.

Uetanabaro, M.; Souza, F. L.; Landgref Filho, P.; Beda, A. F. \& Brandão, R. A. 2007. Anfíbios e répteis do Parque Nacional da Serra da Bodoquena, Mato Grosso do Sul, Brasil. Biota Neotropica 7(3): 279-289.

Valdujo, P. H.; Camacho, A.; Recoder, R. S.; Teixeira, M.; Ghellere, J. M. B; Mott, T.; Nunes, P. M. S.; Nogueira, C. \& Rodrigues, M. T. U. 2011. Anfíbios da Estação Ecológica Serra Geral do Tocantins, região do Jalapão, estados do Tocantins e Bahia. Biota Neotropica 11(1):251-262.

Valdujo, P. H.; Recorder, R. S.; Vasconcellos, M. M. \& Portella, A. S. 2009. Amphibia, Anura, São Desidério western Bahia uplands, northeastern Brazil. Check List 5(4):903-911.

Valdujo, P. H.; Silvano, D. L.; Colli, G. \& Martins, M. 2012. Anuran Species Composition and Distribution Patterns in Brazilian Cerrado, 
a Neotropical Hotspot. South American Journal of Herpetology 7(2):63-78.

Vaz-Silva, W.; Guedes, A. G.; Azevedo-Silva, P. L.; Gontijo, F.

F.; Barbosa, R. S.; Aloísio, G. R. \& Oliveira, F. C. G. 2007.
Herpetofauna, Espora hydroelectric power plant, state of Goiás, Brazil. Check List 3(4):338-345.

ZAR, J. H. 1999. Biostatistical analysis. New Jersey, Prentice Hall. 929p.

Apêndice 1. Lista de exemplares testemunhos das espécies de anuros registradas para o município de Barro Alto, GO entre as estações reprodutivas de 2007 a 2010.

Adenomera aff. marmorata (3540), Adenomera hylaedactyla (3541), Barycholos ternetzi (3526-3527), Chiasmocleis albopunctata (3549, 3561), Dendropsohus cruzi $(3513,3535,3542)$, D. rubicundulus (6029 - 6030), D. minutus (4714-4726), D. nanus (6019 - 6021), D. soaresi (5961-5962), Elachistocleis cf. cesarii (3553), Physalaemus nattereri (3522), Hypsiboas raniceps (3521), H. albopunctatus (35683571), H. goianus (3974-3975), H. lundii (3645-3647), H. paranaiba (6033-6035), Leptodactylus labirynthicus (3664), L. podicipinus (3649, 3660), Leptodactylus cf. furnarius (3642-3644), L. fuscus (5365-5366),
Leptodactylus cf. latrans (3640-3641), Leptodactylus aff. leptodactyloides (6095-6096), L. mystaceus (5364), L. mystacinus (5387), L. syphax (3546), Phyllomedusa azurea (3565-3566), Physalaemus centralis (3528), $P$. cuvieri (3532-3533), Procerathrophys bagnoi (3531, 3554), Pseudis bolbodactyla (3529), Rhinella granulosa (3518-3519), R. schneideri (3563), Scinax constrictus (3515-3516), Scinax fuscomarginatus (3545, 3636), Scinax aff. fuscovarius (3560, 3572), Scinax gr. catharinae (3662), Trachycephalus typhonius $(3520,3646)$. 